\title{
Effect of Indirect Neural Decompression with Oblique Lateral Interbody Fusion Was Influenced by Preoperative Lumbar Lordosis in Adult Spinal Deformity Surgery
}

\author{
Tan Boon Beng ${ }^{1,2}$, Yoshihisa Kotani ${ }^{1}$, Ung Sia ${ }^{3}$, Ivan Gonchar ${ }^{1}$ \\ ${ }^{1}$ Department of Orthopedic Surgery, Steel Memorial Muroran Hospital, Muroran, Japan \\ ${ }^{2}$ Department of Orthopedic Surgery, Faculty of Medicine and Health Science, University Malaysia Sarawak, Kota Samarahan, Malaysia \\ ${ }^{3}$ Department of Orthopaedic Surgery, Sarawak General Hospital, Kuching, Malaysia
}

\begin{abstract}
Study Design: Retrospective clinical study on the indirect decompressive effect of oblique lateral interbody fusion (OLIF) for adult spinal deformity.

Purpose: To evaluate the effect of interbody distraction by OLIF for the treatment of adult spinal deformity.

Overview of Literature: Adult spinal deformity with symptomatic stenosis has been addressed conventionally using a direct posterior decompression approach with fusion. However, stenotic symptoms can also be alleviated indirectly through restoration of intervertebral and foraminal heights and correction of spinal alignment.

Methods: Twenty-eight patients with adult spinal deformity underwent OLIF combined with modified cortical bone trajectory screws at 94 lumbar levels with neuromonitoring. The patients were divided into three groups based on their preoperative lumbar lordosis: group $A,<0^{\circ}$; group $B, 0^{\circ}-20^{\circ}$; and group $C,>20^{\circ}$. The cross-sectional area (CSA) of the thecal sac was measured preoperatively and postoperatively on axial magnetic resonance images. Differences in CSA were evaluated, and the relationship between the CSA extension ratio and preoperative CSA was assessed. Changes in disc height and segmental disc angle were measured from plain radiographs.

Results: OLIFs were performed successfully without neural complications. In group A, the mean CSA increased from $120.6 \mathrm{~mm}^{2}$ preoperatively to $148.5 \mathrm{~mm}^{2}$ postoperatively $(p<0.001)$. The mean CSA for group B increased from $120.1 \mathrm{~mm}^{2}$ preoperatively to $154.4 \mathrm{~mm}^{2}$ postoperatively $(p<0.001)$. Group $C$ had an increase in mean CSA from $114.7 \mathrm{~mm}^{2}$ preoperatively to $160.7 \mathrm{~mm}^{2}$ postoperatively $(p<0.001)$. The mean CSA enlargement ratio was $27.5 \%, 32.1 \%$, and $60.4 \%$ in groups $A, B$, and $C$, respectively. The mean CSA extension ratio was inversely correlated with preoperative CSA.

Conclusions: The effect of indirect neural decompression in adult spinal deformity with OLIF varies with the degree of preoperative lumbar lordosis.
\end{abstract}

Keywords: Adult spinal deformity; Magnetic resonance imaging; Indirect neural decompression; Oblique lateral interbody fusion; Cortical bone trajectory screws

Received Oct 30, 2018; Revised Jan 22, 2019; Accepted Feb 14, 2019

Corresponding author: Tan Boon Beng

Department of Orthopaedic Surgery, Faculty of Medicine and Health Science, University Malaysia Sarawak (UNIMAS), 94300 Kota Samarahan, Sarawak, Malaysia

Tel: +60-168008786, E-mail: jtbb78@yahoo.com

Co-corresponding author: Yoshihisa Kotani

Department of Orthopaedic and Spine Surgery, Steel Memorial Muroran Hospital, Muroran, Hokkaido, Japan

Tel: +81-9052261543, Fax: +81-0143-47-4354, E-mail: yoshi96k@ja2.so-net.ne.jp 


\section{Introduction}

The treatment of adult spinal deformity remains a challenge, despite advances in the medical field. Adult spinal deformity with symptomatic stenosis has been addressed conventionally using a direct posterior decompression approach with fusion. Nevertheless, the surgical options for adult spinal deformity have evolved and now range from limited minimally invasive surgery (MIS) to extensive and demanding osteotomies performed as a single or multistage procedure [1-5]. This evolution continues with the hope of finding a procedure that can address the pathology without causing much collateral damage.

Oblique lateral interbody fusion (OLIF) is a promising MIS procedure that can address the degenerative problems in the spine, including adult spinal deformity [6]. The potential advantages of OLIF compared with other surgical procedures are the ability to indirectly decompress the neural elements by restoring disc height (DH), less risk of direct neural injury, less invasion of the psoas muscle and lumbar plexus, direct visualization of sensory nerves and important anatomical structures, and consistent access to the L4-L5 level in cases with high-riding pelvis [6].

The minimally invasive lateral lumbar interbody fusion (LLIF) technique is able to decompress neural structures by indirect decompression $[7,8]$. However, there are limited data regarding OLIF in adult spinal deformity. The purpose of this study is to evaluate the radiological effect of interbody distraction by the OLIF approach combined with fixation with modified cortical bone trajectory (CBT) screws for the treatment of adult spinal deformity.

\section{Materials and Methods}

\section{Patients}

This was a retrospective clinical study on the indirect decompressive effect of OLIF for adult spinal deformity.
Between January 2013 and March 2015, 28 patients presenting with adult spinal deformity underwent OLIF combined with modified CBT screws at 94 primary lumbar levels with neuromonitoring. This study was approved by the ethical committee at Steel Memorial Muroran Hospital (approval no., J170502).

The number of OLIFs performed at the respective levels was 19 at L1-L2, 26 at L2-L3, 28 at L3-L4, and 21 at L4-L5. A $6^{\circ}$ lordotic-angled, trapezoid-shaped polyetheretherketone cage packed with autologous iliac crest bone graft was placed in all the affected disc spaces. The patients were divided into three groups based on their preoperative lumbar lordosis: group $\mathrm{A}, \angle 0^{\circ}$; group $\mathrm{B}, 0^{\circ}-$ $20^{\circ}$; and group $\mathrm{C},>20^{\circ}$. Table 1 shows the demographic data for the respective groups. Magnetic resonance imaging (MRI) was performed successfully in all patients.

The cross-sectional area (CSA) of the thecal sac was measured preoperatively and postoperatively on $\mathrm{T} 2$ -

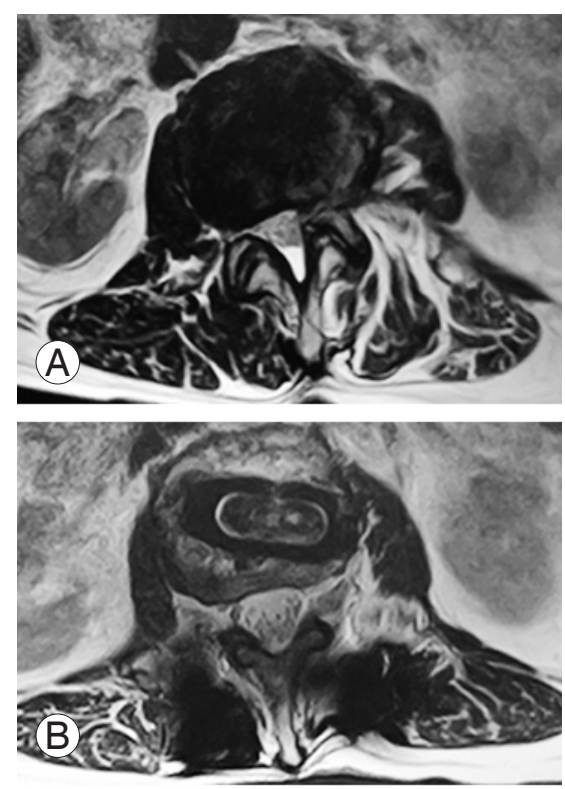

Fig. 1. Preoperative (A) and postoperative (B) magnetic resonance imaging for comparison of cross-sectional area.

Table 1. Patients demographic data

\begin{tabular}{lcccccl} 
Lumbarlordosis & Cases available & Levels fix & $\begin{array}{c}\text { Oblique lateral interbody } \\
\text { fusion level }\end{array}$ & Age (yr) & Body mass index $\left(\mathrm{kg} / \mathrm{m}^{2}\right)$ & Gender \\
$<0$ & 4 & 9.5 & 3.5 & 69.5 & 22.89 & $1 \mathrm{M}, 3 \mathrm{~F}$ \\
$0-20$ & 10 & 9.0 & 3.5 & 75.6 & 21.98 & $10 \mathrm{~F}$ \\
$>20$ & 14 & 6.6 & 3.4 & 74.4 & 22.89 & $2 \mathrm{M}, 12 \mathrm{~F}$ \\
\hline
\end{tabular}

M, male; F, female. 
weighted axial MRIs (Fig. 1). Differences in CSA were analyzed statistically, and the relationship between the CSA extension ratio and that of the preoperative CSA was assessed. The CSA extension ratio was calculated by the formula (postoperative CSA-preoperative CSA)/ preoperative $\mathrm{CSA} \times 100 \%$. Changes in $\mathrm{DH}$ and segmental disc angle (SDA) were measured from plain lateral radiographs.

\section{Measurement of cross-sectional area, disc height, and segmental disc angle}

Based on the MRI, a single axial slice through the center of the disc was used as the comparative measure location in axial views for measurement of CSA. CSA $\left(\mathrm{mm}^{2}\right)$ was measured with Impax software (Agfa-Gevaert Group, Mortsel, Belgium). DH and SDA were measured from plain lateral radiographs of the spine. The distances between the anterior, midpoint, and posterior diameter of the caudal endplate and the cranial endplate were measured to represent DH. SDA was defined as the sagittal Cobb angle between the cranial endplate and the caudal endplate of the affected disc.

\section{Statistical analysis}

Pre- and postoperative CSA, DH, and SDA in the three groups were compared statistically by the paired $t$-test. Correlations between the CSA extension ratio and preoperative CSA and between changes in CSA and other radiological parameters were analyzed using the Pearson correlation coefficient or Spearman rank correlation according to the normality of the parameters assessed. To investigate the factors that affect the CSA extension ratio, regression analysis was performed using the following variables: preoperative CSA, preoperative $\mathrm{DH}$, preoperative SDA, change in DH, and change in SDA. Statistical analysis was performed with SPSS ver. 15.0 (SPSS Inc., Chicago, IL, USA). A $p$-value less than 0.05 was considered to indicate statistical significance in all analyses.

\section{Oblique lateral interbody fusion procedure}

The surgical approach was performed from the concave side of the spinal deformity with the patient positioned in a true lateral decubitus position. Multiple OLIFs were done using a single transverse skin incision. A 3- to $5-\mathrm{cm}$ skin incision at the anterior axillary line, centered over the second highest affected disc level, was made in the lateral abdominal region parallel to the fibers of the external oblique muscle. The external oblique, internal oblique, and transverse abdominal muscles were then dissected along the direction of their fibers. The retroperitoneal space was accessed by blunt dissection, and the peritoneal content was mobilized anteriorly. The psoas muscle and genitofemoral nerve were identified, and the intervertebral disc was exposed through an open corridor between the psoas muscle and the aorta [9], whereas the sympathetic chain and the ureter were mobilize anteriorly. Once the intervertebral disc was exposed, the self-retaining retractor was placed under illumination, and the operative field was ensured. After a portal was made by excising the annulus fibrosis, the disc material, including the cartilaginous endplate, was removed sequentially, and the annulus fibrosis at the opposite side was released completely with caution using a Cobb elevator under fluoroscopic viewing. A trial cage, followed by an appropriate-sized cage filled with autologous bone graft from the iliac crest and synthetic bone substitute as required, was inserted orthogonally in a press-fit fashion into the disc spaces.

Upon completion of the OLIF procedure, the patient was turned to the prone position, and supplemental posterior instrumentation was then placed percutaneously to avoid cage subsidence and loss of the correction [8]. We use a modified CBT screw technique as our supplementary posterior fixation. The screws have a minimum diameter of $5.5 \mathrm{~mm}$, which is larger than that of the originally proposed screws for CBT [10]. Based on our modified CBT technique, the dorsal cortex of the lamina is the insertion point, in contrast to the conventional pedicle screw. The screw is angled toward the superior-anterior angle of the vertebral body on the sagittal plane. The trajectory in the axial plane is either straight or with some degree of divergence with the aid of navigation [11].

\section{Results}

OLIF was performed successfully in all 28 patients without significant neural, vascular, or ureter complications. Five patients had approach-related complications, such as hip flexor weakness, but all resolved spontaneously within 3 months after the operation. The incidence of this hip flexor weakness was $18 \%$. OLIF was performed at a total of 94 lumbar levels. The majority of the levels had success- 
Table 2. Comparison between preoperative and postoperative CSA

\begin{tabular}{lccc}
\multirow{2}{*}{$\begin{array}{l}\text { Lumbar } \\
\text { lordosis }\end{array}$} & \multicolumn{2}{c}{ Mean CSA $\left(\mathrm{mm}^{2}\right)$} & \\
\cline { 2 - 3 } & Preoperative & Postoperative & \\
Group $\mathrm{A}\left(<0^{\circ}\right)$ & 120.6 & 148.5 & $<0.001$ \\
Group B $\left(0^{\circ}-20^{\circ}\right)$ & 120.1 & 154.4 & $<0.001$ \\
Group $\mathrm{C}\left(>20^{\circ}\right)$ & 114.7 & 160.7 & $<0.001$ \\
\hline
\end{tabular}

CSA, cross-sectional area.

Table 3. Correlation between various radiological parameters

\begin{tabular}{lcc} 
Variable & $\begin{array}{c}\text { Correlation } \\
\text { coefficient }\end{array}$ & $p$-value \\
\hline $\begin{array}{l}\text { Preoperative CSA } \\
\text { Change in CSA }\end{array}$ & -0.210 & 0.054 \\
\hline CSA extension ratio & -0.606 & $<0.001$ \\
\hline $\begin{array}{l}\text { Change in CSA } \\
\text { Change in disc height }\end{array}$ & 0.090 & 0.412 \\
\hline Change in segmental disc angle & 0.181 & 0.097 \\
\hline
\end{tabular}

CSA, cross-sectional area.

ful indirect neural decompression, except for five lumbar levels.

In group A, the mean CSA increased from $120.6 \mathrm{~mm}^{2}$ preoperatively to $148.5 \mathrm{~mm}^{2}$ postoperatively $(p<0.001)$. The mean CSA for group B increased from $120.1 \mathrm{~mm}^{2}$ preoperatively to $154.4 \mathrm{~mm}^{2}$ postoperatively $(p<0.001)$. Group C had an increase in mean CSA from $114.7 \mathrm{~mm}^{2}$ preoperatively to $160.7 \mathrm{~mm}^{2}$ postoperatively $(p<0.001)$. The mean CSA enlargement ratio was $2.5 \%, 32.1 \%$, and $60.4 \%$ in groups $\mathrm{A}, \mathrm{B}$, and $\mathrm{C}$, respectively. The overall CSA extension ratio was $45.3 \%$. The mean CSA extension ratio was found to correlate inversely with preoperative CSA $(-0.606)$ using the Spearman rank correlation test. The results are summarized in Tables 2 and 3 .

\section{Discussion}

There are limited studies of OLIF in adult spinal deformity. Despite the many theoretical advantages of OLIF, particularly the effect of indirect neural decompression achieved through the restoration of $\mathrm{DH}$, there were still doubts whether this effect can be achieved and reproduced in adult spinal deformity.

All 28 of our patients with adult spinal deformity were successfully treated with OLIF combined with modified
CBT screws. The effect of indirect neural decompression was analyzed by comparing the preoperative and postoperative MRI results. The overall CSA extension ratio in our study was $45.3 \%$, which is substantially higher than the $8.4 \%$ in the study by Oliveira et al. [7]. This difference is probably due to the additional posterior supplemental fixation that prevents cage subsidence and subsequent loss of correction, in contrast to the stand-alone extreme lateral interbody fusion. In addition, CBT screws have better screw-to-bone purchase and have been proven biomechanically to achieve superior stability compared with traditional pedicle screws $[12,13]$. The CBT technique was associated with lower rates of screw loosening and less frequent loss of correction compared with traditional approaches.

Fujibayashi et al. [6] also reported a lower CSA extension ratio than that in our study. This could be explained by the choice of technique for posterior supplemental fixation. In our study, the patients had OLIF combined with modified CBT screws. This technique has been biomechanically proven to have better insertional torque during pedicle screw insertion $[10,13,14]$. This is a huge advantage, considering the bone quality in this group of patients. Another possible reason is the difference in the choice of subjects in the two studies. The focus of our study was mainly on adult spinal deformity, whereas the other study had a more diversified group of patients with lumbar degenerative disease.

In addition to the effect of indirect neural decompression, OLIF has the advantage of LLIF compared with conventional posterior procedures. Direct neural decompression with posterior procedures is associated with the risks of direct neural injury, epidural hematoma, iatrogenic durotomy, epidural bleeding, and postlaminectomy instability [15-17]. Furthermore, posterior osteotomies are technically demanding and may cause overwhelming collateral damage in this fragile population.

OLIF also allows direct manipulation of the anterior and middle columns, which permits a potentially greater degree of deformity correction compared with manipulation from a posterior approach alone, while minimizing blood loss and overall operative time in patients with adult degenerative scoliosis [18]. A larger cage can be inserted with OLIF than with posterior lumbar interbody fusion or transforaminal lumbar interbody fusion. This may have the advantage of distributing the vertical stress more widely, thereby reducing the incidence of cage 
subsidence, especially in patients with osteoporosis [19]. Therefore, OLIF with modified CBT screws is a promising alternative to the conventional approach to adult spinal deformity.

The purpose of this study was to evaluate the effect of indirect neural decompression with OLIF in adult spinal deformity. We found that the effect of interbody distraction varied with the degree of preoperative lumbar lordosis. There was a higher and significant difference in the CSA extension ratio when the preoperative lumbar lordosis was more than $20^{\circ}$. The degree and rigidity of the adult spinal deformity could be a limiting factor to the ligamentotaxis effect, which is crucial for indirect neural decompression in OLIF.

Our study also showed that the greater the stenosis preoperatively, the greater the improvement in neural decompression. This finding is consistent with the results of the study by Fujibayashi et al. [6]. Therefore, indirect neural decompression is not limited by the severity of spinal stenosis and should be considered an option in place of conventional direct neural decompression.

The indirect neural decompression effect of OLIF was greater at the L2-L3 and L3-L4 disc levels than at other levels. This could be explained by the limitations in approaching the disc and especially in inserting the cage without causing any endplate damage, which may lead to an unsatisfactory cage position and even cage subsidence. At the L1-L2 level, the rib cage may be an obstacle to the approach, whereas the L4-L5 level is more demanding technically due to the iliac crest, especially in patients with high-riding pelvis.

In this series, the incidence of hip flexor weakness was $18 \%$, which is consistent with the reported risk of transient hip flexor weakness following LLIF surgery $[18,20$ 22]. The incidence of hip flexor weakness in the literature ranges from $15 \%$ to $25 \%$. This is probably due to stretching of the iliopsoas muscle during placement of the cage. To minimize this complication, we adopt an approach at the concave side of the spinal deformity, where the iliopsoas muscles are more relaxed. Performing OLIF at various levels using a single transverse skin incision is also possible with this strategy.

Despite the promising results of our study, we must acknowledge the limitations as well. Our study involved a small series of cases, but nevertheless, all the patients were categorized as adult spinal deformity cases. A larger series would truly reflect the advantages and disadvantages of
OLIF combined with modified CBT screws. The numbers of patients in the three groups, based on preoperative lumbar lordosis, were unequal. This was because patients with kyphotic deformity are only a small percentage of the overall population of patients with adult spinal deformity in our clinical practice. Nevertheless, the mean age of the patients was similar in the three groups. There was no documentation and assessment of the bone quality of the patients prior to surgery, which is not routinely performed in our practice.

\section{Conclusions}

The effect of indirect neural decompression in adult spinal deformity with OLIF and modified CBT screws varies with the degree of preoperative lumbar lordosis. Nevertheless, this technique has the potential to control and correct misalignment in adult spinal deformity.

\section{Conflict of Interest}

No potential conflict of interest relevant to this article was reported.

\section{References}

1. Scheufler KM, Cyron D, Dohmen H, Eckardt A. Less invasive surgical correction of adult degenerative scoliosis, part I: technique and radiographic results. Neurosurgery 2010;67:696-710.

2. Scheufler KM, Cyron D, Dohmen H, Eckardt A. Less invasive surgical correction of adult degenerative scoliosis: part II: complications and clinical outcome. Neurosurgery 2010;67:1609-21.

3. Boachie-Adjei O, Ferguson JA, Pigeon RG, Peskin MR. Transpedicular lumbar wedge resection osteotomy for fixed sagittal imbalance: surgical technique and early results. Spine (Phila Pa 1976) 2006;31:48592.

4. Kim YJ, Bridwell KH, Lenke LG, Cheh G, Baldus C. Results of lumbar pedicle subtraction osteotomies for fixed sagittal imbalance: a minimum 5-year followup study. Spine (Phila Pa 1976) 2007;32:2189-97.

5. Watanabe K, Lenke LG, Daubs MD, et al. A central hook-rod construct for osteotomy closure: a technical note. Spine (Phila Pa 1976) 2008;33:1149-55.

6. Fujibayashi S, Hynes RA, Otsuki B, Kimura H, Take- 
moto M, Matsuda S. Effect of indirect neural decompression through oblique lateral interbody fusion for degenerative lumbar disease. Spine (Phila Pa 1976) 2015;40:E175-82.

7. Oliveira L, Marchi L, Coutinho E, Pimenta L. A radiographic assessment of the ability of the extreme lateral interbody fusion procedure to indirectly decompress the neural elements. Spine (Phila Pa 1976) 2010;35:S331-7.

8. Marchi L, Abdala N, Oliveira L, Amaral R, Coutinho E, Pimenta L. Radiographic and clinical evaluation of cage subsidence after stand-alone lateral interbody fusion. J Neurosurg Spine 2013;19:110-8.

9. Barrey C, Ene B, Louis-Tisserand G, Montagna P, Perrin G, Simon E. Vascular anatomy in the lumbar spine investigated by three-dimensional computed tomography angiography: the concept of vascular window. World Neurosurg 2013;79:784-91.

10. Santoni BG, Hynes RA, McGilvray KC, et al. Cortical bone trajectory for lumbar pedicle screws. Spine J 2009;9:366-73.

11. Mobbs RJ. The "medio-latero-superior trajectory technique": an alternative cortical trajectory for pedicle fixation. Orthop Surg 2013;5:56-9.

12. Baluch DA, Patel AA, Lullo B, et al. Effect of physiological loads on cortical and traditional pedicle screw fixation. Spine (Phila Pa 1976) 2014;39:E1297-302.

13. Matsukawa K, Yato Y, Kato T, Imabayashi H, Asazuma $T$, Nemoto K. In vivo analysis of insertional torque during pedicle screwing using cortical bone trajectory technique. Spine (Phila Pa 1976) 2014;39:E240-5.

14. Matsukawa K, Yato Y, Imabayashi H, Hosogane N, Asazuma T, Nemoto K. Biomechanical evaluation of the fixation strength of lumbar pedicle screws using cortical bone trajectory: a finite element study. J Neu- rosurg Spine 2015;23:471-8.

15. Uribe JS, Deukmedjian AR, Mummaneni PV, et al. Complications in adult spinal deformity surgery: an analysis of minimally invasive, hybrid, and open surgical techniques. Neurosurg Focus 2014;36:E15.

16. Lonergan T, Place H, Taylor P. Acute complications following adult spinal deformity surgery in patients aged 70 years and older. J Spinal Disord Tech 2012 Oct [Epub]. https://doi.org/10.1097/ BSD.0b013e3182764a23.

17. Daubs MD, Lenke LG, Cheh G, Stobbs G, Bridwell KH. Adult spinal deformity surgery: complications and outcomes in patients over age 60. Spine (Phila Pa 1976) 2007;32:2238-44.

18. Tempel ZJ, Gandhoke GS, Bonfield CM, Okonkwo DO, Kanter AS. Radiographic and clinical outcomes following combined lateral lumbar interbody fusion and posterior segmental stabilization in patients with adult degenerative scoliosis. Neurosurg Focus 2014;36:E11.

19. Kotwal S, Kawaguchi S, Lebl D, et al. Minimally invasive lateral lumbar interbody fusion: clinical and radiographic outcome at a minimum 2-year followup. J Spinal Disord Tech 2015;28:119-25.

20. Benner B, Ehni G. Degenerative lumbar scoliosis. Spine (Phila Pa 1976) 1979;4:548-52.

21. Anand N, Baron EM, Thaiyananthan G, Khalsa K, Goldstein TB. Minimally invasive multilevel percutaneous correction and fusion for adult lumbar degenerative scoliosis: a technique and feasibility study. J Spinal Disord Tech 2008;21:459-67.

22. Anand N, Rosemann R, Khalsa B, Baron EM. Midterm to long-term clinical and functional outcomes of minimally invasive correction and fusion for adults with scoliosis. Neurosurg Focus 2010;28:E6. 